\title{
LA INVESTIGACIÓN CIENTÍFICA Y EL DESEMPEÑO PROFESIONAL EN LA FORMACIÓN DE TERCER NIVEL EN EL ECUADOR
}

\section{SCIENTIFIC RESEARCH AND PROFESSIONAL PERFORMANCE IN THIRD LEVEL TRAINING IN ECUADOR}

Erick X. López Reyes (exIr@hotmail.com)

Marco Berméo (mbermeo@upse.edu.ec)

Washington Perero (wperero@upse.edu.ec

Víctor Matías (vmatias@upse.edu.ec)

\section{RESUMEN}

La preocupación por la calidad y pertinencia de la educación superior a nivel mundial, ha hecho que el Ecuador adopte e implemente reformas en sus procesos legislativos, administrativos y operativos en materia de educación superior. Empero, el exceso de celo en el cumplimiento de esta tarea ha hecho que se pasen por alto y se obvien algunos principios genéticos básicos de la educación-formación universitaria de tercer nivel (profesional y/o de grado). El presente artículo busca propiciar la necesaria y muy postergada discusión respecto a una realidad que se ha pretendido desconocer y obviar, producto de factores de entropía generados en ópticas y políticas neoliberales, la formación de investigadores profesionales, de nivel uno, en el tercer nivel. En otras palabras, de profesionales de grado (Licenciatura - Ingeniería) cuyo ejercicio estará dedicado en más de un 90\% a actividades de investigación, y que, eventualmente, podrán ingresar a la carrera docente universitaria y al fortalecimiento complementario y progresivo de su perfil profesional de investigador a nivel del postgrado (Maestría y Doctorado Filosofal), en concordancia al mandato y encargo social que han recibido desde sus mismos orígenes las universidades.

Palabras clave: Educación Superior, formación de tercer nivel, investigación profesional y/o de grado.

\section{ABSTRACT}

Concern about the quality and relevance of higher education worldwide, has made Ecuador adopt and implement a series of reforms in their legislative, administrative and operational in higher education processes. In but the overzealous in fulfilling this task has made overlooked and some basic genetic principles of education-university tertiary education (professional and / or grade) is obviated. This article seeks to provide the necessary and very postponed discussion about a reality that has sought to ignore and avoid product of factors entropy generated in optical and neoliberal policies, training of professional researchers, level one, on the third level. In other words professionals (degree - Engineering) whose exercise will be dedicated by more than $90 \%$ to research, and that may possibly enter the university teaching career and complementary and progressive strengthening of their professional profile researcher graduate level (Masters and Doctorate Philosopher), in accordance with the mandate and social order they have received from the very beginning universities.

Key words: Higher education, university education, tertiary education, professional research and / or grade.

Recibido: agosto de 2016 Aprobado: octubre de 2016

\section{Finalidad y funciones sustantivas de la educación/formación de nivel superior.}

"A las puertas del siglo XXI, en medio de un mundo globalizado, la universidad como institución social que tiene la misión de transformar la sociedad buscando el bien de la humanidad y su desarrollo sostenible, a través de la formación de profesionales, la investigación científica y la extensión, tiene el reto social de buscar alternativas que contribuyan a propiciar cambios socio económicos, perfeccionar la organización social y lograr una mejor adaptación a los cambios que se avecinan." (Alemán, 2004) A nivel mundial se reconoce y acepta de forma consensuada, más allá de alguna variación terminológica, la existencia de al menos cuatro funciones sustantivas esenciales que todo centro, entidad o institución educativo-formativa de tercer nivel, grado, o superior, debe cumplir: Docencia (Formación), 
Investigación (Generación), Gestión (Administración) y Vinculación con la comunidad, entorno o contexto (extensión). Estas funciones esenciales, son el sustento y la razón de ser de la vida misma de estos centros educativo-formativos de nivel superior, en torno a los cuales se organiza, o se debería organizar, todo el accionar y razón de ser de estos entes corporativo-institucionales (Mayorga, 1999; Fabre, 2005; Pacheco, 2008; UNESCO, 2009; Andión, 2009).

Desde una perspectiva holística, lógico-operativa, se sustentan en el principio de que el principal patrimonio, activo o capital, por excelencia, de la educación-formativa superior es el conocimiento humano, en todas sus formas y particularidades, más allá del consabido conocimiento científico-técnico, positivista o no positivista. A él recurre -lo gestiona, investiga, aplica y trasfiere por vinculación- en tanto recurso, insumo y materia prima, para el cabal cumplimiento del encargo y rol social, atribuido desde las instituciones, implícita (experticia reconocida) y explícitamente (facultad jurídica), por la sociedad.

Este encargo y rol social que no es otro que el de educar y formar, a nivel superior, el talento humano que dicha sociedad requiere, tanto para dar solución a sus problemas generales y específicos de estabilidad y desarrollo cotidiano (profesionalización ciudadana); así como para atender aquellos procesos que propicien e induzcan su cambio y transformación a futuro (anticipación prospectivo creativa e innovadora), trascendiendo inclusive a aquellos problemas que aún no se avizoran en el corto, mediano o largo plazo (trascendencia predictiva cognitivo generativa).

La educación-formación profesional y técnica de sus ciudadanos (ciudadanía profesionalizada), o mejor aún de su profesionalización cívica, académica y científica, acorde al contexto de sus requerimientos, aspiraciones y potencialidades; es lo que se espera que haga un profesional de nivel superior. Esto es, que aplique de manera pragmática los conocimientos, habilidades y destrezas adquiridas durante su paso por las aulas universitarias, en la identificación y resolución de problemas profesionales propias de su particular campo de estudio y actuación en el cual se formó, y que desde dicha perspectiva cognitiva actúan, suceden, y ocurren al interior del contexto y tejido social. (Delors, 1996; Gradolí, 2015)

En base a las diferentes dimensiones de la cotidianeidad y de las tendencias en prospectiva y proyectiva del desarrollo futuro, la sociedad plantea y encarga a las Instituciones de Educación Superior (IES), la formación del talento humano profesionalizado a nivel superior. $Y$ esto ha sido así desde la génesis misma de las primeras academias y universidades (Ponce, 1974; Ribeiro, 1982; Palacios,1984; Rüegg, 1992; Huff , 1993; Cardini, 1994; Sanz y Bergan, 2002; González, 2006; Pucciarelli y Kaplan, 2016; Buchbinder, s.f.), constituyéndose así, este rol y encargo social a las universidades, en una especie de principio lógico de carácter consuetudinario que define y sustenta las denominadas funciones sustantivas del ser y hacer de las IES.

\section{2.- La Universidad como noción académica y concepto fuente de la educación-formación superior: Ac- tualizando lo esencialmente básico.}

En la actualidad, gracias al desarrollo de la denominada sociedad del conocimiento (Bernal, 2014), vinculada, impulsada y propiciada por el avance científico-tecnológico del accionar académico profesional de las nuevas tecnologías de la comunicación, ha permitido cada vez un mayor y acelerado proceso de producción, acopio e intercambio de información, a nivel de la sistematización y validación de experiencias y diálogo especializado, lo cual de modo sincrónico o diacrónico, ha contribuido a traspasar las barreras de lo imaginable, respecto a la validación, resignificación de los ingentes corpus teóricos y metodológicos disciplinares preexistentes.

No obstante, más allá de toda esta inimaginable realidad, se ha propiciado las más fantásticas iniciativas e inventivas generativas y/o aplicativas a nivel de la producción intelectual, científica, académica y técnica. De las cuales las funciones sustantivas de las IES tampoco están exentas en cuanto se refiere a la gestión operativa procesual de sus particulares y específicos procesos de planificación, ejecución y control, subyace un claro distanciamiento, bien por desconocimiento, omisión o banalización, de lo elemental y esencialmente básico: la lógica académico-científico y profesional de todo este proceso.

Tal situación plantea la urgente necesidad de mirar hacia atrás y recuperar lo básico del principio lógico consuetudinario del rol y encargo social a las universidades, donde principios tan elementales como el hecho de que aparte de todo el avance científico y tecnológico que existe hoy en día, a nivel de paradigmas, teorías, metodologías, técnicas y procesos; el nada simple pero cotidiano hecho de leer, razonar y comunicar, es, ha sido y será siempre la base misma del aprendizaje a nivel superior, así como en otro cualquier nivel.

La lógica académica de la finalidad y de la ruta formativa del talento humano profesionalizado 
(educado y formado) a nivel superior o universitario, que la sociedad y su entorno, natural y/o cultural, requieren y demandan a nivel de sus necesidades (problemas generales y específicos de su adelanto, progreso y desarrollo), sentidas y no sentidas / tangibles o intangibles; bajo cualquier situación de orden paradigmático (teórico metodológico, u otro) que aspire o pretenda organizar y direccionar, en tal o cual sentido, la articulación y ejecución efectiva de las funciones sustantivas de la educación-formación superior.

En el orden de atender con la más alta calidad posible de sus esfuerzos el mandato y encargo social, razón de su existencia, nunca podrá dejar de lado la esencia básica conceptual de la institución social a la cual debe su génesis, y en base a la cual se edifica su actual naturaleza y estructura en tanto entidad académica-científico-formativa, que a decir verdad, más allá de lo formal de su evolución, actualización y cambio, en la esencia misma de su rol de Alma Mater de sociedades y naciones, poco o nada a variado.

$Y$ es que en realidad nada ha cambiado respecto a necesidad y requerimiento del encargo social a ella encomendada, esto es: La formación de talento humano especializado para el ejercicio profesional, el mantenimiento y desarrollo de la cultura, la ciencia, las artes y la técnica (tecnología). De ahí su función y acepción latina de "Madre que nutre" o "Madre nutricia", que alimenta y alienta la vida y el desarrollo de la sociedad a la cual se debe, en franca simbiosis sinérgica. Baste revisar la siguiente definición conceptual de lo que es una Universidad, perfectamente válida y generalizable a cualquier IES, en tanto: “... entidad orgánica o sistema de unidades operativas de enseñanza superior, investigación y creación de cultura científica y humanística.", que asentada en uno o varios lugares llamados campus otorga grados académicos y títulos profesionales a nombre de la nación" (Campos [et.all], 1991).

Se ha considerado necesario redundar respecto a esto, que si bien ya se expresó anteriormente, a efectos de puntualizar y fortalecer el punto de partida del tema que se quiere tratar en el presente texto, muy en particular considerando los ribetes que toda esta situación ha tomado en el Ecuador, donde hoy se busca, contra toda lógica y principio académico superior, ilegítimamente incluso, restringir el "estatus" y condición de investigador, así como el de la docencia superior, a la formación de cuarto nivel, post profesional y/o de postgrado. Haciendo tabula rasa de que la preparación para tales menesteres se logra previamente, incluso, de modo exclusivo en el tercer nivel. Otra cosa es, que no se haya puesto dicho énfasis formativo dentro de la planificación y diseño curricular de las titulaciones correspondientes, muy en particular de aquellas titulaciones profesionales con grados académicos de licenciatura o ingeniería.

Es en el tercer nivel que se debe dar, formar, y evaluar que se concrete la articulación efectiva de la investigación en los diferentes perfiles profesionales, tal cual lo dictamina y exige la norma y lógica académica formativa del talento humano profesionalizado a nivel superior. Cuyo valor genético universal contrasta con la actual creencia administrativo-operativa que impregna la concepción e implementación de la normatividad reglamentaria de la norma jurídica ecuatoriana en materia de educación superior.

No obstante, se debe hacer énfasis en la diferenciación fundamental que existe entre lo que son "Grados Académicos" y "Títulos Profesionales", respecto al tipo de aval o reconocimiento formativo terminal que otorgan, o deberían otorgar, al finalizar el plan curricular de estudios, pensum formativo, curso o programa de titulación. Siendo esto sobre lo que se busca precisamente llamar la atención.

La naturaleza de esta diferenciación básica de estas certificaciones y/o reconocimientos universitarios, cuya esencia misma debe marcar la hoja de ruta a seguir en todo proceso de planificación curricular superior, habitualmente es poco entendido, desconocido, confundido, minimizado o pasado por alto, en "pro" de aspectos más actuales, y novedosos, de la concepción operativa de la gestión o gerencia universitaria.

3.- Certificaciones y Reconocimientos Universitarios: En esencia ¿Al término de la educación-formación de tercer nivel, qué es lo que se reconoce y certifica?.- Títulos Profesionales per se y Títulos profesionales con grado académico.

La formación del talento humano profesionalizado a nivel superior, que la sociedad encomienda a las IES en correspondencia a sus necesidades y problemas generales y específicos de desarrollo, acorde al contexto y entorno, independiente de cualquier eventual desenvolvimiento y curso personal, según las diferentes motivaciones, expectativas y aspiraciones individuales de cada uno de ellos; debe priorizar y mediatizar, de cara al ejercicio y desempeño profesional, así como a garantizar el respectivo mantenimiento, difusión aplicativa, acrecentamiento generativo del conocimiento y los saberes que constituyen su patrimonio, la evidente capacidad para identificar, idear y proponer las soluciones más pertinentes y factibles a dichos problemas, necesidades y demandas que se 
presenten en tal o cual campo del conocimiento humano, incluso de aquellos que aún no están en ciernes. (González, 2006; Alemán, 2008; Vessuri, 2008; Bracamonte, 2015)

Esto lleva a reconocer dos escenarios de actuación, o mejor dicho, de planificación de la gestión académica curricular formativa, en correspondencia a los dos tipos genéricos de titulaciones que la universidad y las otras IES, otorgan, esto es: Titulaciones Profesionales específicas, y Titulaciones Profesionales con Grado Académico, pero proyectivas, es decir con un derivativo hacia la vida académica o perfeccionamiento profesional a nivel de la ciencia y la filosofía (Pacheco, 2000 y 2008).

Estos reconocimientos universitarios, o de nivel superior, certifican o avalan los estudios cursados, y determinan desde ya, o mejor dicho, deberían determinar, la eventual y potencial ruta formativa a seguir del profesional superior, en cuanto una eventual necesidad de actualización de conocimientos, fortalecimiento y perfeccionamiento del perfil profesional alcanzado tras la titulación/ graduación. Ya sea por actualización de conocimientos y/o continuidad de estudios en un cuarto nivel de instrucción educativo-formativa, educación continúa post profesional y/o de postgrado.

Pacheco Prado (2000), en el Ecuador, indicó que la naturaleza tipológica y la finalidad última de estas titulaciones suelen ser confundidas, creándose "una serie de dificultades no solamente personales para quienes ostentan esos títulos y grados, sino que en diversas ocasiones se registran problemas a nivel interinstitucional, tanto internamente como a nivel internacional, cuando se trata de equiparar estos reconocimientos."

Como plantea el autor antes mencionado y, parafraseando el contenido textual semántico del concepto, como aparece en los diferentes diccionarios y enciclopedias de lengua española, "tener una profesión supone que una persona conoce de un arte, oficio, habilidad o facultad y que de manera expresa declara ponerlos al servicio de los demás" (...) "Las profesiones como una forma de servicio universitario constituyeron, desde el comienzo, el fundamento de la organización universitaria". Así mismo, el ejercicio y el desempeño profesional, "un servicio de la persona a la sociedad", se concibe y entiende, que es "el medio a través del cual la persona, el individuo, desarrolla la actividad en provecho suyo. Es decir, su patrimonio que está constituido por un conjunto de conocimientos y habilidades, los vende a los demás, quienes a su vez deben reconocerle un pago por la venta de estos servicios." (Op.cit.)
Bajo estos postulados lo que se espera de todo profesional, y muy en especial de aquellos formados a nivel superior, independiente del nivel y/o campo de su formación, es que sea capaz de detectar, identificar, delimitar y solucionar problemas profesionales en el área del conocimiento en la cual estudió y se formó. En otras palabras que aplique en la práctica social, con criterio profesional, los conocimientos, habilidades, aptitudes, capacidades y destrezas adquiridas a nivel metodológico, procedimental, actitudinal e instrumental, en el campo del saber de su especialidad, presto a responder a través de su ejercicio y desempeño profesional, como medio de vida y subsistencia.

Todo esto necesariamente al margen, y aparte de los serios cuestionamientos que a fines del siglo pasado gestó el actual movimiento transformador de la educación-formación superior, o universitaria, cuyo punto de partida fue marcado por la agenda de debate establecida y desarrollada en el marco de la "Conferencia Mundial sobre Educación Superior", celebrada en París en octubre de 1998; y que de forma indefectible llevó a la adopción de una nueva "cultura universitaria".

En todo caso, al ser las IES, muy en particular las universidades, los centros donde se forman y prepararan los profesionales de nivel superior que requiere la sociedad, es su responsabilidad ineludible e intransferible, acorde al entorno, contexto y momento histórico en que se desenvuelve, la de certificar y abalizarlas competencias profesionales generales de desempeño, a más de testar y calificar aquellas particulares de la especialidad cognitivo-operativa del ejercicio específico de tal o cual profesión/titulación (González, 2006; Alemán, 2008; Pacheco 2008)

Estos aspectos generales del ejercicio profesional no son otros que: el identificar problemas en su campo de formación laboral (diagnosis); en base a la educación-formación adquirida, idear y proponer la solución más pertinente y factible al mismo; conforme a ello ejecutar un proceso básico y elemental de recolección (investigación) de datos (empíricos y/o teóricos) que sustenten su postura profesional frente a la solución del problema identificado (Tesis); sistematizar y analizar la información obtenida a efectos de abstraer y elaborar conclusiones y/o recomendaciones pertinentes y válidas respecto a la solución pragmática y efectiva del problema. Finalmente deberá, según sea el caso, emitir una síntesis textual u oral informativa de lo efectuado (informe), absolviendo cualquier duda o inquietud que este generase (defensa) en la o las personas que requirieron de sus servicios (Bernal, 2014; Isaza, 2015).

Es esto, a más de los evidentes componentes 
cognitivos (teórico-metodológicos), procedimentales (operativo-instrumentales), y actitudinales (axiológicos), lo que las IES deben evaluar y certificar, poseen sus profesionales al término de cualquier carrera. Caso contrario no estarán cumpliendo con el encargo social que la sociedad y el Estado les ha confiado. De ahí que se estile que al culminar sus estudios superiores, el profesional egresado elabore una demostración práctica, a modo de ejercicio de reconocimiento y certificación de competencia genérica macro laboral, un pequeño trabajo de diagnosis, diseño, ejecución, informe y defensa, de lo que se espera hará durante el resto de su vida como profesional: Identificar y solucionar de manera pertinente y factible problemas profesionales en su campo de formativo de actuación.

Las IES, las universidades, en tanto entidad académica formativa deben certificar y reconocer, $y$ de hecho es lo que se supone hacen a través de la correspondiente emisión y otorgamiento de los denominados Títulos Profesionales y Grados Académicos Universitarios, mismos que responden a diferentes lógicas, realidades y finalidades de ejercicio y desempeño, conforme los distintos objetivos, objetos, y metas proyectivas contempladas en tal o cual ruta o proceso educativo-formativo de este tipo.

Se debe acotar que aunque las actuales tendencias, paradigmas, preocupaciones, cuestionamientos, etc., que contextualizan, matizan y particularizan corológica y cronológicamente hablando, la problemática actual de la educación-formación de nivel superior; volviendo la mirada hacia atrás, regresando a la base esencial de la génesis universitaria conforme el ya discutido mandato y encargo social recibido de parte del conglomerado humano que la genera y acoge, debe responder con calidad y pertinencia. Se debe tener en claro que se está hablando de dos tipos de reconocimiento y certificaciones: Títulos profesionales per se, tales como Arquitecto, Biólogo, Arqueólogo, etc., y Títulos Profesionales con grado académico, a nivel de licenciatura o ingeniería, como serían las licenciaturas en arquitecto, en biología, arqueología, etc., o las ingenierías de todo tipo orientativo (Pacheco, 2000 y 2008).

Los primeros facultan única y exclusivamente para el ejercicio pleno de la profesión adquirida, en tanto los segundos, a más de esto, direccionarían, o mejor dicho deberían hacerlo, el ingreso a la academia, bien como docente y/o investigador, tras dos años de ejercicio profesional comprobado. En todo caso, ambas situaciones responden a la lógica de la potencial y eventual trayectoria, que conforme sus expectativas, gustos, motivaciones y capacidades, el profesional de tercer nivel, superior o universitario, decide hacer una carrera estrictamente basada en lo profesional, o sienta la necesidad motivacional de prestar además su contingente de servicio a la academia y/o a la Ciencia. Lamentablemente, esta premisa de génesis orientativa, tan elemental y básica de planificación universitaria, se ignora y se pasa por alto, por desconocimiento y/o falta de formación académica, por parte de quienes tienen la más noble tarea de direccionar y ejecutar la planificación de la educación-formación superior, y mucho menos es comunicada a los aspirantes a ingresar en las correspondientes carreras que se ofertan a este nivel. Al menos es lo que sucede en el Ecuador.

En todo caso, valga agregar para concluir este punto, que en el orden de la actualización, fortalecimiento y mejoramiento constante del perfil profesional, de cara a la diversificación de los escenarios de su actuación como tal, y conforme dichos procesos, es que existen los denominados cursos y/o programas de Educación Continua y/o Postgrado, que en su conjunto definen la educación-formación de cuarto nivel (Pacheco, 2000 y 2008), de los cuales en el presente trabajo se toparán únicamente, en tanto su lógica genética de finalidad última, aquellos aspectos relativos a la investigación en tanto herramienta para y del ejercicio profesional en general y/o específico, y la formación profesional de quienes la investigación es la esencia básica, general y específica de su ejercicio profesional, que es el objetivo central del presente texto.

Antes de continuar se quisiera aclarar, que de ninguna manera la posición que aquí se sostiene tenga alguna especie de animadversión o que se quiera estigmatizar de algún modo la formación de cuarto nivel, más bien todo lo contrario, lo que se busca es hacer notar que para que esta pueda desarrollarse de forma efectiva, se hace necesario recuperar la lógica consuetudinaria básica de la finalidad y función de la formación superior del tercer nivel respecto al rol de la investigación en la formación del talento humano que la sociedad, el contexto y la coyuntura socio-histórica demandan precisamente para tales fines, y que continúan ampliando, fortaleciendo y complementando en el cuarto nivel.

\section{4.- La investigación científica como instrumento del ejercicio y la prác- tica profesional superior Vs. La for- mación profesional como académi- co e investigador de un profesional en el nivel de grado.}

Sin el ánimo de entrar a discutir desde el punto 
de vista coneptual qué es investigación, se puede mencionar que de acuerdo a las definiciones que presenta la Real Academia Española (RAE) sobre la palabra investigar (vocablo que tiene su origen en el latín investigare), este verbo se refiere "al acto de llevar a cabo estrategias para descubrir algo. También permite hacer mención al conjunto de actividades de índole intelectual y experimental de carácter sistemático, con la intención de incrementar los conocimientos sobre un determinado asunto"

En todo caso resulta evidente que se trata de una actividad humana, orientada a la obtención de nuevos conocimientos y su aplicación para la solución a problemas o interrogantes (significados. com) de cualquier tipo o carácter, y no únicamente "científico".

"En ese sentido, puede decirse que una investigación está determinada por la averiguación de datos o la búsqueda de soluciones para ciertos inconvenientes. Cabe destacar que una investigación, en especial en el plano científico, es un proceso sistemático (se obtiene información a partir de un plan preestablecido que, una vez asimilada y examinada, modificará o añadirá conocimientos a los ya existentes), organizado (es necesario especificar los detalles vinculados al estudio) y objetivo (sus conclusiones no se amparan en un parecer subjetivo, sino en episodios que previamente han sido observados y evaluados)." (definicion. de)

De ahí entonces que la "acción o efecto de investigar" que entraña conceptualmente el término "investigación", "puede tener varios objetivos: buscar soluciones a problemas puntuales, desentrañar las causas de una problemática social, desarrollar un nuevo componente de uso industrial, etc., no obstante, su finalidad es siempre la misma: conocer la realidad, descubrir algo, entender un proceso, encontrar un resultado." (significados.com)

Tomando en cuenta todo lo hasta ahora argumentado, es fácilmente entendible entonces, el por qué la investigación sea, o deba ser considerada, una herramienta de trabajo más del ejercicio efectivo de un profesional, independientemente de la orientación formativo-cognitivo-operativa adquirida en las IES; que debe necesariamente ser tratada como un eje formativo transversal de toda propuesta curricular del tercer nivel, no tanto porque el profesional a cuya formación se oriente vaya a desempeñarse necesariamente como investigador profesional, como podría ser el caso de los biólogos, naturalistas, entomólogos, historiadores, sociólogos, antropólogos o de los arqueólogos, solo por citar unos casos.
Si tal es la situación en cuanto aquellas profesiones avaladas por el título profesional per se, con mucha mayor razón hay que considerar estos ejes transversales de formación en cuanto aquellas profesiones con grado académico (licenciaturas e ingenierías), cuyo "plus" radica precisamente en que la concesión, reconocimiento y certificación del grado correspondiente en este nivel de educación superior, faculta a quien lo posea y ostente, a un eventual ingreso a la academia como docente y/o investigador, hacia el desempeño profesional como investigador en su respectivo campo de formación, así como a un ulterior desarrollo y perfeccionamiento como tal a nivel de postgrado (Maestría y Doctorado).

De hecho, obedeciendo a esta lógica académica de la educación-formación superior, es que un verdadero y efectivo trabajo de grado de tercer nivel permita, entre otras cosas:

- Comprobar y certificar la capacidad y competencia profesional de los graduandos de una carrera de grado.

- Permita poner en práctica y, a través de ella, pulir las capacidades investigativas del novel profesional, que hecho va a desempeñarse profesionalmente como investigador, y eventualmente fortalecerse como tal a nivel de maestrías y doctorados.

- Aportar significativamente al conocimiento científico técnico de una profesión, al mediar entre un problema y su correspondiente solución.

- Idear soluciones pertinentes y factibles a problemas específicos dentro de un ámbito profesional.

- Ensayar las aptitudes y competencias analítico-criticas, reflexivo-constructivas, sintético-demostrativas de procesos argumentativos e intelectuales como profesional (textualidad y oralidad).

Lamentablemente está elemental norma y principio básico esencial de la planificación y gerencia académica superior, al parecer se ha olvidado, o al menos diluido en gran medida, en los actúales procesos de "mejoramiento" continuo, al punto que pareciera ser inadmisible, incluso incompatible y hasta impensable, el hecho de que se pueda hablar de la formación profesional de investigadores profesionales (profesionales cuyo ejercicio profesional al término del tercer nivel es precisamente el hacer investigación, sea esta básica o aplicada, en sus correspondientes campos cognitivo-operativos de formación). De hecho, uno de los legados de la aplicación de la lógica no académica, mercantilista del neoliberalismo, ha sido la de correr el umbral de la madurez intelectual formativa de los estudiantes, muy en especial de quienes serán profesionales universitarios; dando 
en el post doctorado, lo que se debió dar en el doctorado, en el doctorado lo que le correspondía a la maestría, en la maestría lo que se supone da el tercer nivel, y en este lo que se supone debió haber dado la educación media.

La norma lógica, académico formativa, en general destina la formación de tercer nivel a la identificación y solución de problemas profesionales en los correspondientes campos de formación y ejercicio profesional. A nivel de las Maestrías se busca perfeccionar el perfil del investigador, licenciados y/o ingenieros, ya fogueado en tales menesteres, muy en particular en el manejo de hipótesis de primer grado. Orientándolos y dotándoles de aquellos componentes cognitivos, procedimentales y actitudinales que les permitan hacer contribuciones, creativos y/o innovadores, a corpus teóricos o metodológicos preestablecidos, a través del manejo de hipótesis de segundo grado. Los Doctorados (Ph.D) apuntan básicamente a lo mismo, pero el nivel de exigencia formativa apunta a la concepción generativa de una nueva teoría y/o metodología, a través del manejo de hipótesis de tercer grado, y del dominio del pensamiento y del discurso cognitivo filosofal, considerado el nivel más alto del pensamiento académico-científico de la humanidad (Pacheco,2008). Si bien las verdades inmutables no existen, empero ahí están.

Devolviendo la esencia misma, su majestuosidad, a la formación de tercer nivel en el Ecuador, respecto a las diferentes titulaciones que se oferta y otorga en la IES, se podrá entonces garantizar un desarrollo pleno de estos ciudadanos profesionalizados a nivel del cuarto nivel, tanto postprofesional como de postgrado.

\section{5.- A manera de conclusión, una ur- gente recomendación.}

Para finalizar, los autores del presente texto quieren dejar especificado, que con lo hasta aquí planteado, no se está cuestionando de ningún modo la validez y pertinencia de los aspectos y procesos que en materia de innovación, evaluación y certificación de la calidad y pertinencia de la educación superior en el Ecuador se han venido, se están, y de seguro se continuaran implementando. Más bien por el contrario, han considerado necesario y oportuno, considerando precisamente el escenario actual, la urgente necesidad de llamar la atención, enfatizando la validez y pertinencia de actualidad, sobre algunos principios básicos de la génesis, lógica y esencia académica que pareciera habérseles olvidado, por omisión, desconocimiento, o simple falta de formación académica, de quienes piensan, diseñan y textualizan normativamente la legislación educativa superior en el

\section{Ecuador.}

Si bien la preocupación por mejorar los actuales procesos formativos y de gestión académica universitaria en nuestro país ha concitado el interés de otros elementos y claustros universitarios del país (Cielo, Ospina y vega, 2016), mucho más allá de que sus planteamientos vayan en correspondencia a la preocupación mundial, que fue definiendo una agenda que cuestionaba, propugnaba y replanteaba el cambio consustancial de muchos de los procesos metodológicos, operativos y gestión, que por entonces las IES desarrollaban, al considerárselos caducos, anacrónicos y sin sentido, al tenor de las nuevas tendencias, enfoques y paradigmas tecnológicos y de la educación-formación superior. Estos están centrados en cuestiones de carácter más normativo y operativo, descuidando y obviando temas esenciales como los hasta aquí brevemente tratados. Muy en particular el hecho de que la condición de investigador profesional, así como una formación para el eventual ingreso a la academia superior como docente se logra, o debería lógraselo, es a nivel de la formación de tercer nivel, ya que esta es la que prepara para la identificación y resolución de problemas puntuales y/o generales de desarrollo, en el ámbito social cotidiano, y en prospectiva, como parte del ejercicio profesional laboral. Un profesional se desempeña, labora y trabaja precisamente de cara a esta actuación, y en base a ello es que crea, genera, mantiene, transmite, y acrecienta el conocimiento científico de su profesión, tanto en lo empírico como en lo teórico.

El Ecuador ha procurado acortar distancias en estos aspectos, procurando dar un salto cuántico y sustancial en su propio sistema educativo a todo nivel, si bien se lo está logrando, empero se nota así mismo un creciente alejamiento y desvinculación de la esencia misma del encargo y mandato social a ella encomendada, al concentrarse en demasía en el cumplimiento y acumulación burocrática de muchos criterios, indicadores y evidencias de papel. Que no necesariamente conducen a la tan pregonada acumulación, gestión y acrecentamiento del conocimiento humano y la formación de los profesionales que la sociedad en general demanda en la solución práctica de sus problemas más generales y específicos de desarrollo, conforme sus particulares contextos históricos, culturales, sociopolíticos, científicos, tecnológicos, etc., etc. De ahí que no sea todavía tarde como para volver la mirada atrás, recuperar e incorporar con sentido de actualidad, lo básico y esencial de la formación académica, profesional y científica de la educación superior. Reposicionando así, a la investigación científica como instrumento del ejercicio y la práctica de todo profesio- 
nal (títulos profesionales), así como el verdadero significado y esencia operativa de la formación de grado (títulos profesionales con grado académico)

\section{Bibliografía}

Alemán P A, El papel de la universidad en el proceso de formación para la economía solidaria. Revista Virtual Universidad Católica del Norte 2008 1-15. Disponible en: http://www.redalyc.org/articulo.oa?id=194220359003. Fecha de consulta: 15 de julio de 2016.

Andión M, La Universidad Nodo como Modelo Universitario. Revista de difusión Casa del Tiempo № 242009 22-25. Universidad Autónoma de México. Disponible en: http://www.uam.mx/difusion/casadeltiempo/24_iv_oct_2009/casa_del_ tiempo_elV_num24_22_25.pdf

Bernal C A, Metodología de la investigación. Para administración, economía, humanidades y ciencias sociales. 3ra Edic. Pearson Educación. 2014. México

Bracamonte $\mathrm{R}$, La responsabilidad social de la Universidad. Revista Científica Multidisciplinaria Adastra. Centro de Estudios de Posgrado e Investigación (CEPI), Universidad San Francisco Xavier de Chuquisaca. 2015 Vol. 6 Nº 42015 3739. Bolivia.

Buchbinder P, La Universidad: Breve introducción a su evolución histórica. Universidad Nacional del Litoral I UNLVIRTUAL. PDF s.f. Disponible en: http://www.unlvirtual.edu.ar/wp-content/recursos/ La_universidad_breve_evolucion_historica.pdf

Cardinl F. [et all.], Universidades de Europa. Anaya, Grandes Obras. ISBN 978-84-8162-988-0. 1994.

Campos R C; De la Garza Vizcaya E; Ortiz Segura y Bustos, Evaluación cualitativa en la educación superior: cinco estudios de caso / México, D.F.: Limusa; Noriega Editores, 1991 . ISBN 96818-4172-7

Cielo, Cristina; Ospina, Pablo; Vega, Cristina. Ed. Reforma y renacimiento: Conversaciones docentes sobre la reforma educativa superior en Ecuador. Universidad Andina Simón Bolívar. Fundación Hernán Malo.Corporación Editora Nacional. Quito. 2016.

definicion.de, Definición de investigación. Diccionario On line. Disponible en; : Definición de investigación - Qué es, Significado y Concepto http:// definicion.de/investigacion/\#ixzz4EsQ3zat5
Delors $\mathrm{J}$ [et. All.], La Educación encierra un tesoro. Informe a la UNESCO de la Comisión Internacional sobre la Educación Superior para el Siglo XXI. 1996 Santillana Ediciones UNESCO.

Fabre Batista G C, Las funciones sustantivas de la universidad y su articulación en un departamento docente. Universidad Agraria de La Habana, Cuba. Ponencia presentada en el V Congreso Internacional Virtual de Educación, 7-27 de Febrero de 2005. Disponible en: http://sedici.unlp.edu.ar/ bitstream/handle/10915/24694/Documento_completo.pdf?sequence $=1$

González Cuevas O M, El Concepto de Universidad, México: Universidad Autónoma Metropolitana Azcapotzalco. 2006 Disponible en: http://www.scielo.org.mx/scielo. php? script $=$ sci_nlinks\& $\mathrm{ref}=1549967 \& \mathrm{pi}-$ $\mathrm{d}=S 1405-1435200900030001200011 \& \operatorname{lng}=e s$

Gradolí A, Resumen del Informe a la UNESCO (Jacques De Lors). Material de clases de la Asignatura PROBLEMES I CORRENTS CONTEMPORANIS EN EDUCACIÓ. Abril de 2015. Disponible en: http://www.neurofilosofia. $\mathrm{com} / . . . /$ Resumen-de-LA-EDUCACION-ENCIERRA-UN-TESORO.pdf

Huff T E, The Rise of Early Modern Science: Islam, China, and the West. Cambridge: Cambridge University Press. 978-0521529945. 1993

Isaza Zapata G M, La formación en investigación desde el método: Un análisis preliminar. En: Revista Fundación Universitaria Luis Amigó. Vol. 2, No. 1, pp. 106-117, enero-junio 2015. ISSN 23823410. Medellín - Colombia.

Mayorga R, Los desafíos a la universidad latinoamericana en el siglo XXI. Revista Iberoamericana de Educación No 21 (1999) pp 25-40 Septiembre - Diciembre. Publicación monográfica cuatrimestral editada por la Organización de Estados lberoamericanos (OEI). Disponible en: http://rieoei. org/rie21a02.htm

Palacios A, Universidad y democracia. 1984 Buenos Aires: Ediciones MNR.

Pacheco Prado L, Los Reconocimientos Universitarios: Los títulos y los Grados. Ponencia presentada en el XI Encuentro Nacional de Planeamiento Universitario. Realizado en la Escuela Politécnica del Ejercito (ESPE). Quito-2000- Octubre

Pacheco Prado L, LA UNIVERSIDAD: Desafíos en la gestión académica. Cap. 5to.- PROFESIO- 
NES, TÍTULOS Y GRADOS, pp. 47-54. PUCE. 2008 Quito. Ecuador

Ponce A, Educación y lucha de clases. 1974 Buenos Aires: Cartago.

Pucciarelli F y Kaplan A, Competition and Strategy in Higher Education: Managing Complexity and Uncertainty, Business Horizons, Volume 59. 2016

Ribeiro, D, La universidad necesaria. México: Universidad Autónoma de México. 968-58-02637. 1982

Rüegg W, Foreword. The University as a European Institution. En: A History of the University in Europe. Vol. 1: Universities in the Middle Ages. Cambridge University Press. 1992 ISBN 0-52136105-2, pp. XIX-XX

Sanz N y Bergan S (eds.), The Heritage of European Universities, Council of Europe. 2002 ISBN 92-871-4960-7.

significados.com, Significado de investigación. Diccionario On line. Disponible en: http://www.significados.com/investigacion/

UNESCO, Conferencia Mundial sobre Educación Superior", celebrada en París en octubre de 1998.

UNESCO, Conferencia Mundial sobre la Educación Superior - 2009: La nueva dinámica de la educación superior y la investigación para el cambio social y el desarrollo. Comunicado del 8 de julio de 2009. Sede de la UNESCO, París, 5-8 de julio.

Vessuri He (ed.), El movimiento de responsabilidad social de la universidad: una comprensión novedosa de la misión universitaria. Revista educación y Sociedad. Nueva Época. Año 13. № 2. Sep-2008. Instituto Internacional de UNESCO para la Educación Superior en América Latina y el Caribe (IESALC). 